\title{
Erratum
}

\section{Insurance Rebates, Incentives and Primary Care in Australia}

\author{
Luke B. Connelly and James R.G. Butler
}

The Geneva Papers (2013) 38, 181. doi:10.1057/gpp.2012.50

Correction to: The Geneva Papers (2012) 38(4) 745-762. doi: 10.1057/gpp.2012.40

Table 1 appearing on page 752 of the article appeared incorrectly as

Table 1 Summary of effects of increase in subsidy on gross (market) price, net price, quantity traded and quantity of services bulk-billed

\begin{tabular}{|c|c|c|c|c|c|c|c|c|}
\hline & \multicolumn{4}{|c|}{ Competition } & \multicolumn{4}{|c|}{ Monopoly } \\
\hline & $p_{g}$ & $p_{n}$ & $q$ & $B B$ & $p_{g}$ & $p_{n}$ & $q$ & $B B$ \\
\hline (a) & $+\Delta p_{g}=\Delta s$ & $\mathrm{nc}$ & $\mathrm{nc}$ & + & $+\Delta p_{g}=\Delta s$ & $\mathrm{nc}$ & $\mathrm{nc}$ & + \\
\hline (b) & $+\Delta p_{g}<\Delta s$ & $-p_{n}^{\prime}=0$ & + & + & $+\Delta p_{g}<\Delta s$ & $-p^{\prime}{ }_{n}=0$ & + & + \\
\hline (c) & $+\Delta p_{g}<\Delta s$ & $-p^{\prime} n>0$ & + & $\mathrm{nc}$ & $+\Delta p_{g}>\Delta s$ & $-p^{\prime}{ }_{n}>0$ & + & nc \\
\hline
\end{tabular}

Notes: These results assume that supply is not perfectly price inelastic and is not backward-bending and that transactions costs are zero; (a) pre- and post-change equilibria characterised by bulk-billing; (b) pre-change equilibrium not characterised by bulk-billing but post-change equilibrium characterised by bulk-billing; (c) neither pre- nor post-change equilibrium characterised by bulk-billing; BB= quantity of services bulk-billed; nc $=$ no change; $+=$ increase, $-=$ decrease.

The above table should have appeared as follows:

Table 1 Summary of effects of increase in subsidy on gross (market) price, net price, quantity traded and quantity of services bulk-billed

\begin{tabular}{|c|c|c|c|c|c|c|c|c|}
\hline & \multicolumn{4}{|c|}{ Competition } & \multicolumn{4}{|c|}{ Monopoly } \\
\hline & $p_{g}$ & $p_{n}$ & $q$ & $B B$ & $p_{g}$ & $p_{n}$ & $q$ & $B B$ \\
\hline (a) & $\begin{array}{c}+ \\
\Delta p_{g}=\Delta s\end{array}$ & $\mathrm{nc}$ & $\mathrm{nc}$ & + & $\begin{array}{c}+ \\
\Delta p_{g}=\Delta s\end{array}$ & $\mathrm{nc}$ & $\mathrm{nc}$ & + \\
\hline (b) & $\begin{array}{c}+ \\
\Delta p_{g}<\Delta s\end{array}$ & $\begin{array}{c}- \\
p_{n}^{\prime}=0\end{array}$ & + & + & $\begin{array}{c}+ \\
\Delta p_{g}<\Delta s\end{array}$ & $\begin{array}{c}- \\
p_{n}^{\prime}=0\end{array}$ & + & + \\
\hline (c) & $\begin{array}{c}+ \\
\Delta p_{g}<\Delta s\end{array}$ & $\stackrel{-}{p_{n}^{\prime}>0}$ & + & $\mathrm{nc}$ & $\begin{array}{c}+ \\
\Delta p_{g}<\Delta s\end{array}$ & $p_{n}^{\prime}>0$ & + & $\mathrm{nc}$ \\
\hline
\end{tabular}

Notes: These results assume that supply is not perfectly price inelastic and is not backward-bending and that transactions costs are zero.

(a) pre- and post-change equilibria characterised by bulk-billing

(b) pre-change equilibrium not characterised by bulk-billing but post-change equilibrium characterised by bulkbilling

(c) neither pre- nor post-change equilibrium characterised by bulk-billing

$\mathrm{BB}=$ quantity of services bulk-billed

$\mathrm{nc}=$ no change

$+=$ increase $;-=$ decrease 